\title{
Vibrational-Chemical Kinetics in Mars Entry Problems
}

\author{
E.V. Kustova ${ }^{*}, 1$, E.A. Nagnibeda ${ }^{1}$ and I. Armenise ${ }^{2}$ \\ ${ }^{1}$ Saint Petersburg State University, Department of Mathematics and Mechanics, 28 Universitetsky pr, 198504 Saint \\ Petersburg, Russia \\ ${ }^{2}$ CNR-IMIP (Consiglio Nazionale delle Ricerche, Istituto di Metodologie Inorganiche e dei Plasmi), Via Amendola \\ 122/D, 70126 Bari, Italy
}

\begin{abstract}
The paper deals with kinetic theory methods modelling of reacting gas flows near spacecrafts entering the Mars atmosphere. For mixtures containing $\mathrm{CO}_{2}$ molecules, the complete kinetic scheme including all vibrational energy transitions, dissociation, recombination and exchange chemical reactions is proposed. For the prediction of gas dynamic parameters and heat transfer to the surface of a spacecraft, a detailed approach taking into account state-to-state $\mathrm{CO}_{2}$ vibrational and chemical kinetics as well as multi-temperature approaches based on quasi-stationary vibrational distributions are used. A more accurate but complicated and time consuming state-to-state model is applied for the numerical simulation of a one-dimensional flow in a boundary layer near the entering body surface. More simple quasistationary three-temperature, two-temperature and one-temperature approaches are used for the numerical study of a 2-D viscous shock layer under entry conditions. The vibrational distributions near the surface are far from the local vibrational and chemical equilibrium and a noticeable difference is found between the values of $\mathrm{CO}_{2}$ vibrational-specific energies at the surface obtained by means of the state-to-state and quasi-stationary approaches. At the same time, for all considered approaches, the kinetic model for vibrational distributions and chemical reactions has a weak influence on the heat transfer to the non-catalytic vehicle surface.
\end{abstract}

Keywords: $\mathrm{CO}_{2}$, Mars entry, multi-temperature models, state-to-state model.

\section{INTRODUCTION}

For the accurate prediction of gas dynamic parameters on the trajectory of nonexpendable space vehicles in their entry into the Mars atmosphere and heat transfer to the vehicle surfaces, non-equilibrium vibrational and chemical kinetics in mixtures containing $\mathrm{CO}_{2}$ should be taken into account. For a large part of the descending trajectory at the altitudes about 40-60 km, the relaxation times of kinetic processes become comparable to the mean time of variation of gas dynamic parameters. Therefore, for mathematical modeling of a flow, the gas dynamic equations should be coupled to the equations for non-equilibrium $\mathrm{CO}_{2}$ kinetics. The important up-to-date problem is the development of adequate $\mathrm{CO}_{2}$ kinetics models and their incorporation into the computational fluid dynamics (CFD) schemes.

The kinetic theory makes it possible to derive closed sets of governing equations of a flow under different nonequilibrium conditions and elaborate calculation procedures for transport coefficients and reaction rates which are included in equations. However, till now, only simplified one-temperature or two-temperature $\mathrm{CO}_{2}$ models [1-4] have been used in numerical simulations. These models do not describe the complex $\mathrm{CO}_{2}$ kinetics well and can lead to considerable errors in predicted values of flow parameters.

*Address correspondence to this author at the Saint Petersburg State University, Department of Mathematics and Mechanics, 28 Universitetsky pr, 198504 Saint Petersburg, Russia; Tel: +7 81242841 98;

Fax: +7 81242869 44; E-mail: kustova@math.spbu.ru
Indeed, in the one-temperature approach the gas is assumed to be in the thermal equilibrium, and only non-equilibrium chemical reactions take place in a mixture. The twotemperature $\mathrm{CO}_{2}$ model is based on introducing a single vibrational temperature for all three vibrational modes of $\mathrm{CO}_{2}$ molecules.

More rigorous multi-temperature [5-9] and state-to-state $[10,11]$ models of $\mathrm{CO}_{2}$ kinetics take into account real structure of carbon dioxide molecules and include to the kinetic scheme intra- and inter-mode vibrational energy exchanges as well as VT vibrational-translational energy transitions. Based on the rigorous kinetic theory algorithms proposed in $[7,10]$, transport properties in dissociating $\mathrm{CO}_{2}$ flows behind shock waves have been evaluated [9]. In these studies, calculations have been performed for the flow parameters found previously from simplified fluid dynamic equations. The most accurate state-to-state models are very detailed and computationally expensive for the numerical simulations of viscous non-equilibrium flows, especially for the transport terms evaluation because a huge number of equations for vibrational level populations of various $\mathrm{CO}_{2}$ modes and state dependent transport coefficients which should be considered. And even simpler multi-temperature models can be complicated for applications, if the vibrational temperatures are introduced for each mode of anharmonic $\mathrm{CO}_{2}$ vibrations and several test cases are needed.

A self-consistent three-temperature description of the three-component $\mathrm{CO}_{2} / \mathrm{CO} / \mathrm{O}$ mixture suitable for applications and providing satisfactory accuracy has been 
proposed in [12] on the basis of the kinetic theory methods using the relations between relaxation times of different $\mathrm{CO}_{2}$ vibrational energy exchanges; its generalization for the fivecomponent five-temperature $\mathrm{CO}_{2} / \mathrm{CO} / \mathrm{O}_{2} / \mathrm{C} / \mathrm{O}$ mixture is proposed in [13]. The model takes into account various mechanisms of $\mathrm{CO}_{2}$ vibrational relaxation and gives the expressions for transport coefficients which may be implemented into the viscous flow solvers. In $[14,15]$, the five-component reacting mixture $\mathrm{CO}_{2} / \mathrm{CO} / \mathrm{O}_{2} / \mathrm{C} / \mathrm{O}$ was studied and the accurate transport algorithms were implemented directly into CFD solver for simulation of a 2D flow in a viscous shock layer near spacecrafts entering the Mars atmosphere. The flows near space vehicles Mars Sample Return Orbiter (MSRO), Mars Express and blunt bodies of different shape for various free stream conditions in the cases of fully-catalytic and non-catalytic vehicle surfaces were studied on the basis of this model.

The objectives of the paper are the following. The first goal is to propose a complete kinetic scheme for a fivecomponent $\mathrm{CO}_{2} / \mathrm{CO} / \mathrm{O}_{2} / \mathrm{C} / \mathrm{O}$ mixture flow taking into account all vibrational energy transitions and main chemical reactions. This general scheme can be applied for any nonequilibrium $\mathrm{CO}_{2}$ flow, and can be simplified under various flow conditions. The second task is to specify the rate coefficients of non-equilibrium processes included to the reduced kinetic scheme used for flow simulations discussed in the paper. The next objective is to elaborate closed selfconsistent macroscopic flow descriptions in the state-to-state and multi-temperature approaches and to derive corresponding governing equations. Finally, we aim to study 1D and 2D non-equilibrium $\mathrm{CO}_{2}$ flows using different models and to analyze the influence of the kinetic model on gas dynamic parameters and transport properties on the descending trajectory under the Mars entry conditions.

The originality of the present paper is in comparison of non-equilibrium vibrational-chemical $\mathrm{CO}_{2}$ kinetics and heat transfer evaluated on the basis of the state-to-state and multitemperature approximations. It is worth mentioning that the vibrational specific state-to-state mechanism for a carbon dioxide mixture has been developed in our previous work [11], and here it is applied for the specific test case. Existing multi-temperature 2D shock layer flow results [14, 15], are used to define the boundary layer edge conditions for the state-to-state simulations performed in this study.

\section{MECHANISMS OF VIBRATIONAL CHEMICAL RELAXATION}

AND

Mars atmosphere consists of $95.32 \%$ carbon dioxide, $2.7 \%$ nitrogen, $1.6 \%$ argon, and small amount of admixtures: oxygen, carbon monoxide, water vapor. For Mars entry problems, there is a considerable increase in the amount of $\mathrm{CO}_{2}$ dissociation products, $\mathrm{CO}$ and $\mathrm{O}$.

$\mathrm{CO}_{2}$ is a linear triatomic molecule. In the ground electronic state it has three vibrational modes: symmetric stretching mode with frequency $v_{1}$, doubly degenerated bending mode $\left(v_{2}\right)$, and asymmetric stretching mode $\left(v_{3}\right)$. Its vibrational energy can be described as [16]

$$
\varepsilon_{v_{1} v_{2} v_{3}}=\sum_{k=1}^{3} \omega_{k}^{e}\left(v_{k}+\frac{d_{k}}{2}\right)+\sum_{k=1}^{3} \sum_{j \geq k}^{3} x_{k j}^{e}\left(v_{k}+\frac{d_{k}}{2}\right)\left(v_{j}+\frac{d_{j}}{2}\right)+x_{l l}^{e} l^{2}+\ldots
$$

where $v_{m}, m=1,2,3$ are the vibrational levels of $\mathrm{CO}_{2}$ symmetric, bending and asymmetric modes respectively, $l$ is the additional quantum number describing the projection of the momentum of bending vibrations onto the axis of the molecule, $d_{k}$ characterize the degeneration $\left(d_{1}=d_{3}=1\right.$ whereas $d_{2}=2$ ). The terms of higher order in the above expansion are small and can be neglected in this study. The vibrational energy of diatomic molecules is modelled by Morse anharmonic oscillator.

In the present work, we do not take into account electronic excitation of atoms and molecules as well as ionization. Moreover, we do not include processes involving nitrogen to the kinetic scheme. These assumptions certainly lead to noticeable limitations of the proposed model, and we plan to incorporate these missing processes at the next step of our work.

For diatomic molecules, there are two main mechanisms of vibrational relaxation, namely, $\mathrm{VV}$ exchanges of vibrational quanta and VT transitions of vibrational energy to the translational one. Since $\mathrm{CO}_{2}$ molecule has multiple vibrational modes, there are several additional relaxation channels. To present the kinetic scheme, we first introduce intra-mode vibrational transitions $\mathrm{VV}_{m}$ :

$$
\begin{aligned}
& \mathbf{V V}_{1}: C O_{2}\left(v_{1}, v_{2}^{l}, v_{3}\right)+C O_{2}\left(w_{1}, w_{2}^{m}, w_{3}\right) \rightleftharpoons \\
& C O_{2}\left(v_{1} \pm 1, v_{2}^{l}, v_{3}\right)+C O_{2}\left(w_{1} \mp 1, w_{2}^{m}, w_{3}\right), \\
& \mathbf{V V}_{2}: C O_{2}\left(v_{1}, v_{2}^{l}, v_{3}\right)+C O_{2}\left(w_{1}, w_{2}^{m}, w_{3}\right) \rightleftharpoons \\
& C O_{2}\left(v_{1}, v_{2}^{l \pm 1} \pm 1, v_{3}\right)+C O_{2}\left(w_{1}, w_{2}^{m \mp 1} \mp 1, w_{3}\right), \\
& \mathbf{V V}_{3}: C O_{2}\left(v_{1}, v_{2}^{l}, v_{3}\right)+C O_{2}\left(w_{1}, w_{2}^{m}, w_{3}\right) \rightleftharpoons \\
& C O_{2}\left(v_{1}, v_{2}^{l}, v_{3} \pm 1\right)+C O_{2}\left(w_{1}, w_{2}^{m}, w_{3} \mp 1\right), \\
& \mathbf{V V}_{\mathrm{O} 2}: O_{2}(v)+O_{2}(w) \rightleftharpoons O_{2}(v \pm 1)+O_{2}(w \mp 1), \\
& \mathbf{V V}_{\mathrm{CO}}: C O(v)+C O(w) \rightleftharpoons C O(v \pm 1)+C O(w \mp 1) .
\end{aligned}
$$

The next relaxation mechanism is due to vibrationaltranslational transitions in each mode $\mathrm{VT}_{m}$ :

$$
\begin{aligned}
& \mathbf{V T}_{1}: C O_{2}\left(v_{1}, v_{2}^{l}, v_{3}\right)+M \rightleftharpoons C O_{2}\left(v_{1} \pm 1, v_{2}^{l}, v_{3}\right)+M, \\
& \mathbf{V T}_{2}: C O_{2}\left(v_{1}, v_{2}^{l}, v_{3}\right)+M \rightleftharpoons C O_{2}\left(v_{1}, v_{2}^{l \pm 1} \pm 1, v_{3}\right)+M, \\
& \mathbf{V T}_{3}: C O_{2}\left(v_{1}, v_{2}^{l}, v_{3}\right)+M \rightleftharpoons C O_{2}\left(v_{1}, v_{2}^{l}, v_{3} \pm 1\right)+M, \\
& \mathbf{V T}_{\mathrm{O} 2}: O_{2}(v)+M \rightleftharpoons O_{2}(v \pm 1)+M, \\
& \mathbf{V T}_{\mathrm{CO}}: C O(v)+M \Leftrightarrow C O(v \pm 1)+M,
\end{aligned}
$$

$\mathrm{M}$ stands for the partner in a collision.

A particular feature of polyatomic molecules relaxation is the existence of inter-mode $\mathrm{VV}_{m-k}$ exchanges, in our case within $\mathrm{CO}_{2}$ molecule:

$$
\begin{aligned}
& \mathbf{V V}_{1-2}: C O_{2}\left(v_{1}, v_{2}^{l}, v_{3}\right)+M \Leftrightarrow C O_{2}\left(v_{1} \pm 1, v_{2}^{l} \mp 2, v_{3}\right)+M,( \\
& \mathbf{V V}_{2-3}: C O_{2}\left(v_{1}, v_{2}^{l}, v_{3}\right)+M \Leftrightarrow C O_{2}\left(v_{1}, v_{2}^{l \pm 1} \pm 3, v_{3} \mp 1\right)+M,(12) \\
& \mathbf{V V}_{1-2-3}: C O_{2}\left(v_{1}, v_{2}^{l}, v_{3}\right)+M \rightleftharpoons C O_{2}\left(v_{1} \pm 1, v_{2}^{l \pm 1} \pm 1, v_{3} \mp 1\right)+M,(
\end{aligned}
$$

and between molecules of different chemical species: 
$\mathbf{V V}_{3-\mathrm{Co}}: \mathrm{CO}_{2}\left(v_{1}, v_{2}^{l}, v_{3}\right)+\mathrm{CO}(w) \rightleftharpoons \mathrm{CO}_{2}\left(v_{1}, v_{2}^{l}, v_{3} \mp 1\right)+\mathrm{CO}(w \pm 1)$,

$\mathbf{V V}_{2-\mathrm{Co}}: \mathrm{CO}_{2}\left(v_{1}, v_{2}^{l}, v_{3}\right)+C O(w) \rightleftharpoons \mathrm{CO}_{2}\left(v_{1}, v_{2}^{l \pm 1} \mp 3, v_{3}\right)+C O(w \pm 1)$,

$\mathbf{V V}_{1-2-\mathrm{CO}}: \mathrm{CO}_{2}\left(v_{1}, v_{2}^{l}, v_{3}\right)+\mathrm{CO}(w) \Leftrightarrow \mathrm{CO}_{2}\left(v_{1} \pm 1, v_{2}^{l \pm 1} \pm 1, v_{3}\right)+\mathrm{CO}(w \mp 1),(16$

$\mathbf{V V}_{1-\mathrm{O}_{2}}: \mathrm{CO}_{2}\left(v_{1}, v_{2}^{l}, v_{3}\right)+\mathrm{O}_{2}(w) \rightleftharpoons \mathrm{CO}_{2}\left(v_{1} \mp 1, v_{2}^{l}, v_{3}\right)+O_{2}(w \pm 1),(17$

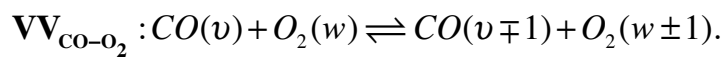

Finally, among state-specific chemical reactions we consider dissociation--recombination:

$\mathrm{CO}_{2}\left(v_{1}, v_{2}, v_{3}\right)+M \rightleftharpoons \mathrm{CO}(w)+O+M$,

$\mathrm{CO}(v)+\mathrm{M} \rightleftharpoons \mathrm{C}+\mathrm{O}+\mathrm{M}$,

$\mathrm{O}_{2}(v)+\mathrm{M} \rightleftharpoons \mathrm{O}+\mathrm{O}+\mathrm{M}$

and exchange reactions:

$\mathrm{CO}_{2}\left(v_{1}, v_{2}, v_{3}\right)+\mathrm{O} \rightleftharpoons \mathrm{CO}(v)+\mathrm{O}_{2}(w)$,

$\mathrm{CO}(v)+\mathrm{O} \rightleftharpoons \mathrm{O}_{2}(w)+C$,

$\mathrm{CO}(v)+\mathrm{CO}(w) \rightleftharpoons \mathrm{CO}_{2}\left(v_{1}, v_{2}, v_{3}\right)+C$

Processes (1)-(24) represent the complete kinetic scheme for a five-component $\mathrm{CO}_{2} / \mathrm{CO} / \mathrm{O}_{2} / \mathrm{C} / \mathrm{O}$ mixture without electronic excitation and ionization. The rates of these processes may differ by several orders of magnitude, which allows neglecting some transitions under particular physical conditions and provides a basis for the development of multi-temperature models.

\section{RATE COEFFICIENTS FOR VIBRATIONAL TRANSITIONS AND CHEMICAL REACTIONS}

In this section, we introduce the rate coefficients for the processes included to reduced kinetic scheme used further for the flow simulations.

One of the problems arising in the modelling of vibrational kinetics for polyatomic molecules is the lack of data on the rate coefficients of different kinds of vibrational energy transitions within and between modes. The diverse experimental results describe only several vibrational transitions between the lowest states (see [1, 17-20]). Unfortunately these data are not sufficient for the description of transitions between highly located states and for the modelling of $\mathrm{VV}$ transitions. The majority of theoretical models are based on the so called SSH-theory [21, 22] which gives only qualitative behaviour of transition probabilities and can be applied for the moderate temperature range. To have confidence on the choice of a model, the rate coefficients should be validated against corresponding experimentally measured values or semi-classical and quantum mechanical trajectory calculations (see [23-26]). We propose the following procedure for the calculation of the transition rates [12]: the rate coefficients of vibrational energy transitions between the lowest vibrational states are computed using the experimental data [20], the remaining rate coefficients (for higher states) are calculated on the basis of the SSH theory [21] modified for polyatomic molecules.

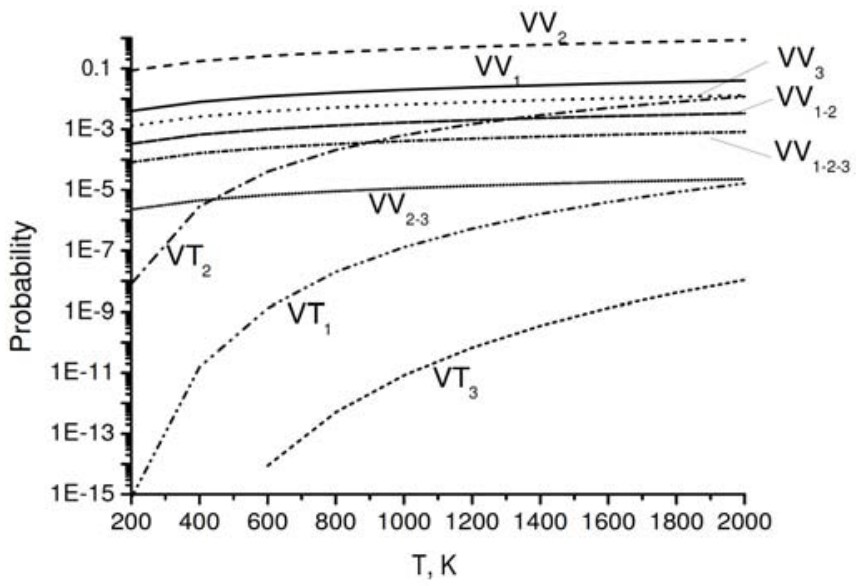

Fig. (1). Probabilities of vibrational energy transitions between the lowest states, the SSH theory.

In Fig. (1), probabilities of vibrational energy transitions between the lowest states calculated on the basis of the SSH theory are reported. One can notice that whereas the probabilities of intra-mode transitions are close to each other, other probabilities differ significantly. Moreover, it is seen that the symmetric mode relax mainly through $\mathrm{VV}_{1-2}$ exchanges and not through $\mathrm{VT}_{1}$ processes. The asymmetric mode relax through inter-mode $\mathrm{VV}_{2-3}, \mathrm{VV}_{1-2-3}$ transitions since the probability of $\mathrm{VT}_{3}$ transitions is low. Therefore, we keep only $\mathrm{VT}_{2}$ transitions as the main channel of VT relaxation in $\mathrm{CO}_{2}$.

On the basis of the SSH theory, we obtain the following expressions for the rate coefficients of the most relevant vibrational transitions:

$$
\begin{aligned}
& V T_{2}: k_{v_{2}+1 \rightarrow v_{2}}^{M}=k_{010 \rightarrow 000}^{M}\left(v_{2}+1\right), \\
& V T_{C O, O_{2}}: k_{v+1 \rightarrow 0}^{M}=k_{1 \rightarrow 0}^{M}(v+1), \\
& V V_{2-3}: k_{v_{2}+3, v_{3} \rightarrow v_{2}, v_{3}+1}^{M}=k_{030 \rightarrow 001}^{M} \frac{\left(v_{2}+1\right)\left(v_{2}+2\right)\left(v_{2}+3\right)\left(v_{3}+1\right)}{3 !}, \\
& V V_{1-2-3}: k_{v_{1}+1, v_{2}+1, v_{3} \rightarrow v_{1}, v_{2}, v_{3}+1}^{M}=k_{110 \rightarrow 001}^{M}\left(v_{1}+1\right)\left(v_{2}+1\right)\left(v_{3}+1\right), \\
& V V_{3-C O}: k_{v_{3}+1 \rightarrow v_{3}}^{w \rightarrow w+1}=k_{001 \rightarrow 000}^{0 \rightarrow 1}\left(v_{3}+1\right)(w+1), \\
& V V_{2-C O}: k_{v_{2}+3 \rightarrow v_{2}}^{w \rightarrow w+1}=k_{030 \rightarrow 000}^{0 \rightarrow 1} \frac{\left(v_{2}+1\right)\left(v_{2}+2\right)\left(v_{2}+3\right)(w+1)}{3 !}, \\
& V V_{1-2-C O}: k_{v_{1}+1, v_{2}+1 \rightarrow v_{1}, v_{2}}^{w \rightarrow w+1}=k_{110 \rightarrow 000}^{0 \rightarrow 1}\left(v_{1}+1\right)\left(v_{2}+1\right)(w+1) .
\end{aligned}
$$

For the more accurate calculation of the rate coefficients $k_{010 \rightarrow 000}^{M}, k_{1 \rightarrow 0}^{M}, k_{030 \rightarrow 001}^{M}$, and so on, we propose to use experimental data from [20]. The rate coefficient for a transition $\gamma$ can be connected to the corresponding relaxation time by an approximate expression

$$
k_{\gamma}=\frac{k T}{p \tau_{\gamma}}
$$

where $k$ is the Boltzmann constant, $T$ is the temperature, $p$ is the pressure in atmospheres, and $\tau_{\gamma}$ is the characteristic time of the process $\gamma . \operatorname{In}[20], \tau_{\gamma}$ is calculated as 
$\log _{10}\left(p \tau_{\gamma}\right)=A_{0}+A_{1} T^{-1 / 3}+A_{2} T^{-2 / 3}$.

Coefficients $A_{n}$ for the different processes and $\mathrm{M}=\mathrm{CO}_{2}$ are reported in Table $\mathbf{1}$.

Table 1. Coefficients $A_{n}$ for various transitions.

\begin{tabular}{|c|c|c|c|}
\hline Process & $\boldsymbol{A}_{\mathbf{0}}$ & $\boldsymbol{A}_{\mathbf{1}}$ & $\boldsymbol{A}_{\mathbf{2}}$ \\
\hline \hline $\mathrm{VT}_{2}$ & -10.327 & 57.31 & -156.7 \\
\hline $\mathrm{VT}_{C O}$ & -12.820 & 155.91 & -450.5 \\
\hline $\mathrm{VV}_{2-3}, \mathrm{VV}_{1-2-3}$ & -12.662 & 88.87 & -272.5 \\
\hline $\mathrm{VV}_{1-2-C O}, \mathrm{VV}_{2-C O}$ & -10.708 & 69.94 & -203.3 \\
\hline $\mathrm{VV}_{3-C O}$ & -9.144 & 31.91 & -103.5 \\
\hline
\end{tabular}

The rate coefficients of backward transitions are connected to the rate coefficients of forward transitions by the detailed balance principle. For vibrational-translational transitions in each mode $\mathrm{VT}_{m}$ or inter-mode $\mathrm{VV}_{m-k}$ exchanges, it reads:

$k_{v_{1^{\prime}}, v_{2^{\prime}}, v_{3^{\prime}} \rightarrow v_{1}, v_{2}, v_{3}}^{M}=k_{v_{1}, v_{2}, v_{3} \rightarrow v_{1^{\prime}}, v_{2^{\prime}}, v_{3^{\prime}}}^{M} \frac{v_{2}+1}{v_{2^{\prime}}+1} \exp \left(\frac{\varepsilon_{v_{1^{\prime} v^{\prime}, v_{3^{\prime}}}}-\varepsilon_{v_{1} v_{2} v_{3}}}{k T}\right)$

For inter-molecular VV transitions we have:

$k_{v_{1^{\prime}}, v_{2^{\prime}}, v_{3^{\prime}} \rightarrow v_{1}, v_{2}, v_{3}}^{w^{\prime} \rightarrow w}=$

$k_{v_{1}, v_{2}, v_{3} \rightarrow v_{1^{\prime}}, v_{2^{\prime}}, v_{3^{\prime}}}^{w \rightarrow w^{\prime}} \frac{v_{2}+1}{v_{2^{\prime}}+1} \exp \left(\frac{\varepsilon_{v_{1^{\prime}, v^{\prime} v_{3^{\prime}}}}+\varepsilon_{w^{\prime}}^{c}-\varepsilon_{v_{1} v_{2} v_{3}}-\varepsilon_{w}^{c}}{k T}\right)$.

In these expressions, $v_{2}+1$ is the statistical weight connected to the degeneration of the bending mode, $\varepsilon_{w}^{c}$ is the vibrational energy of diatomic species $c$.

Let us consider the rate coefficients of state-specific chemical reactions. For the dissociation rate coefficient of $\mathrm{CO}_{2}$ in reaction (19) we use the generalization of the Treanor-Marrone model [27] for three-atomic molecules:

$k_{v_{1} v_{2} v_{3}, d i s s}^{M}=\frac{Z_{v i b r}^{C O_{2}}(T)}{Z_{v i b r}^{C O_{2}}(-U)} \exp \left(\frac{\varepsilon_{v_{1} v_{2} v_{3}}}{k}\left(\frac{1}{T}+\frac{1}{U}\right)\right) k_{d i s s}^{e q, M}$.

Here

$Z_{v i b r}^{C O_{2}}(T)=\sum_{v_{1} v_{2} v_{3}}\left(v_{2}+1\right) \exp \left(-\frac{\varepsilon_{v_{1} v_{2} v_{3}}}{k T}\right)$

is the equilibrium vibrational partition function of $\mathrm{CO}_{2}$, $k_{\text {diss }}^{e q, M}$ is the thermal equilibrium dissociation rate coefficient of $\mathrm{CO}_{2}, U$ is the parameter describing preferential character of dissociation. The rate coefficient for recombination can be expressed with the use of the detailed balance principle:

$k_{r e c, v_{1} v_{2} v_{3}}^{M}(T)=k_{v_{1} v_{2} v_{3}, d i s s}^{M}\left(\frac{m_{C O_{2}}}{m_{C O} m_{O}}\right)^{3 / 2}(2 \pi k T)^{-3 / 2} h^{3}\left(v_{2}+1\right) \frac{Z_{r o t}^{C O}(T)}{Z_{r o t}^{C O}} \times$ $\times \exp \left(-\frac{\varepsilon_{v_{1} v_{2} v_{3}}+\varepsilon^{C O_{2}}-\varepsilon_{w}^{C O}-\varepsilon^{C O}-\varepsilon^{O}}{k T}\right)$,

$h$ is the Planck constant, $Z_{r o t}^{\mathrm{CO}_{2}}, Z_{r o t}^{\mathrm{CO}}$ are the rotational partition functions for $\mathrm{CO}_{2}$ and $\mathrm{CO}$ molecules, $\varepsilon^{c}$ is the formation energy for $c$ species (for homonuclear molecules like $\mathrm{O}_{2}, \varepsilon^{c}=0$ ).

For the dissociation and recombination rate coefficients of diatomic molecules, using the Treanor-Marrone model we obtain

$k_{v, d i s s}^{c, M}=\frac{Z_{v i b r}^{c}(T)}{Z_{v i b r}^{c}(-U)} \exp \left(\frac{\varepsilon_{v}^{c}}{k}\left(\frac{1}{T}+\frac{1}{U}\right)\right) k_{d i s s}^{e q, c, M}, \quad c=C O, O_{2}$

$k_{r e c, v}^{C O, M}(T)=k_{v, \text { diss }}^{C O, M}\left(\frac{m_{C O}}{m_{C} m_{O}}\right)^{3 / 2}$

$(2 \pi k T)^{-3 / 2} h^{3} Z_{r o t}^{C O}(T) \exp \left(-\frac{\varepsilon_{v}^{C O}+\varepsilon^{C O}-\varepsilon^{C}-\varepsilon^{O}}{k T}\right)$,

$k_{\text {rec }, v}^{O_{2}, M}(T)=k_{v, \text { diss }}^{O_{2}, M}\left(\frac{2}{m_{O}}\right)^{3 / 2}(2 \pi k T)^{-3 / 2} h^{3} Z_{\text {rot }}^{O_{2}}(T) \exp \left(-\frac{\varepsilon_{v}^{O_{2}}-2 \varepsilon^{O}}{k T}\right)$

with the vibrational partition function for diatomic species

$Z_{v i b r}^{c}(T)=\sum_{v} \exp \left(-\frac{\varepsilon_{v^{c}}}{k T}\right)$

The state-to-state rate coefficients of forward exchange reactions also can be related to the equilibrium Arrhenius rate coefficients using the non-equilibrium factor, see [28]. In this paper the following expression is proposed for $k_{v, f, r}$ (" $r$ " denotes one of the reactions (22)-(24); " $f$ " corresponds to the forward reaction; for $\mathrm{CO}_{2}, v$ stands for the set of quantum numbers $\left(v_{1}, v_{2}, v_{3}\right)$ :

$k_{v, f, r}=k_{f, r}^{e q}(T) Z v(T, U)$

$Z_{v}(T, U)$ is the non-equilibrium factor specified by the activation energy of $r$-th reaction, $E_{a, r}$

$Z_{v}(T, U)=\left\{\begin{array}{ll}F(T, U) \exp \left(-\frac{E_{a, r}}{k U}\right) \exp \left(\frac{\varepsilon_{v}^{c}}{k}\left(\frac{1}{T}+\frac{1}{U}\right)\right), & \varepsilon_{v}^{c}<E_{a, r}, \\ F(T, U) \exp \left(\frac{E_{a, r}}{k T}\right), & \varepsilon_{v}^{c}>E_{a, r},\end{array}\right\}$

normalizing function $F(T, U)$ is given by

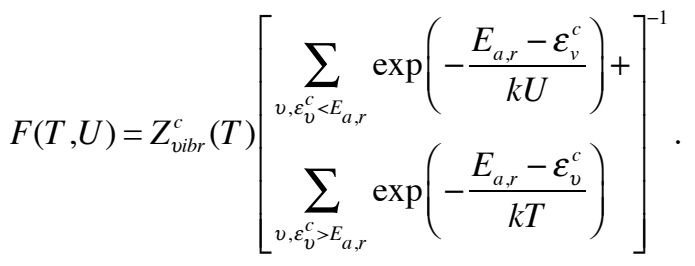


Note that if $E_{a, r}$ approaches to the dissociation energy, the rate coefficients for exchange reactions (39) reduce to the dissociation rate coefficients (34) or (36).

The backward reaction rate coefficients for the reactions (22)-(24) $k_{v, b, r}$ are found applying the detailed balance principle:

$k_{v, b,}=k_{f,}\left(\frac{m_{\mathrm{CO}_{2}} m_{O}}{m_{C O} m_{O_{2}}}\right)^{3 / 2}$

$\frac{Z_{\text {rot }}^{C O_{2}}}{Z_{r o t}^{C O} Z_{r o t}^{O_{2}}} \exp \left(-\frac{\varepsilon_{v_{1} v_{2} v_{3}}-\varepsilon_{v}^{C O}-\varepsilon_{w}^{O_{2}}+\varepsilon^{C O_{2}}+\varepsilon^{O}-\varepsilon^{C O}}{k T}\right)$,

$k_{v, b,}=k_{f,}\left(\frac{m_{C O} m_{O}}{m_{O_{2}} m_{C}}\right)^{3 / 2}$

$\frac{Z_{r o t}^{C O}}{Z_{r o t}^{O_{2}}} \exp \left(-\frac{\varepsilon_{v}^{C O}-\varepsilon_{w}^{O_{2}}+\varepsilon^{C O}+\varepsilon^{O}-\varepsilon^{C}}{k T}\right)$,

$k_{v, b,}=k_{f,}\left(\frac{m_{\mathrm{CO}}^{2}}{m_{\mathrm{CO}_{2}} m_{C}}\right)^{3 / 2}$

$\frac{\left(Z_{r o t}^{C O}\right)^{2}}{Z_{\text {rot }}^{C O}} \exp \left(-\frac{\varepsilon_{v}^{C O}+\varepsilon_{w}^{C O}-\varepsilon_{v_{1} v_{2} v_{3}}+2 \varepsilon^{C O}-\varepsilon^{C O_{2}}-\varepsilon^{C}}{k T}\right)$.

Table 2. Thermal equilibrium rate coefficients for reactions (19)-(22). Mc-Kenzie model.

\begin{tabular}{|c|c|}
\hline Reaction & $\boldsymbol{k}_{f, r} \mathbf{c m}^{3} \mathbf{m o l e}^{-1} \mathbf{s}^{-1}$ \\
\hline \hline$(19)$ & $7.2 \cdot 10^{11} T^{1 / 2} \exp (-36850 / T)$ \\
\hline$(20)$ & $3.5 \cdot 10^{12} T^{1 / 2} \exp (-92800 / T)$ \\
\hline$(21)$ & $0.77 \cdot 10^{17} T^{-1 / 2} \exp (-59400 / T)$ \\
\hline$(22)$ & $0.61 \cdot 10^{12} \exp (-17600 / T)$ \\
\hline
\end{tabular}

Table 3. Thermal equilibrium rate coefficients for reactions (19)-(24). Park model.

\begin{tabular}{|c|c|}
\hline Reaction & $\boldsymbol{k}_{f, r} \mathbf{m}^{3} \mathbf{m o l e}^{-1} \mathbf{s}^{-1}$ \\
\hline \hline$(19)$ & $6.9 \cdot 10^{15} T^{-1.5} \exp (-63275 / T)$ \\
\hline$(20)$ & $2.3 \cdot 10^{14} T^{-1} \exp (-129000 / T)$ \\
\hline$(21)$ & $2.0 \cdot 10^{15} T^{-1.5} \exp (-59500 / T)$ \\
\hline$(22)$ & $2.1 \cdot 10^{7} \exp (-27800 / T)$ \\
\hline$(23)$ & $3.9 \cdot 10^{7} T^{-0.18} \exp (-69200 / T)$ \\
\hline$(24)$ & $2.33 \cdot 10^{3} T^{0.5} \exp (-65710 / T)$ \\
\hline
\end{tabular}

Thermal equilibrium rate coefficients of chemical reactions can be found using various models. In Tables $\mathbf{2}$ and $\mathbf{3}$ the rate coefficients are given according to the Mc-Kenzie [29] and Park [30] models. For dissociation reactions $M=\mathrm{CO}_{2}$. More recent experimental data on dissociation rate coefficients in $\mathrm{CO}_{2}$ mixtures can be found in [31, 32].

Thus we have all required information on the rate coefficients for vibrational transitions and chemical reactions contributing to the simplified kinetic mechanism applied in the results section.

\section{STATE-TO-STATE DESCRIPTION OF VIBRATIONAL-CHEMICAL KINETICS}

The most detailed state-to-state model of vibrationchemical kinetics and transport properties is needed under the condition of strong vibrational and chemical coupling:

$\tau_{\text {tr }}<\tau_{\text {rot }}<<\tau_{\text {vibr }}<\tau_{\text {react }}<<\theta$,

where $\tau_{t r}, \tau_{\text {rot }}, \tau_{v i b r}$, and $\tau_{\text {react }}$ are respectively the characteristic times of translational, rotational, vibrational and chemical relaxation, $\theta$ is the time of gas-dynamic parameters variation. The closed set of macroscopic parameters consists of vibrational level populations of $\mathrm{CO}_{2}$ $n_{v_{1} v_{2}^{l} v_{3}}$ and diatomic species $n_{w}^{c}$, number densities of atoms $n_{c}$, macroscopic flow velocity $v$, and gas temperature $T$.

The general set of governing equations has the form

$\frac{d \rho}{d t}+\rho \nabla \cdot \mathbf{v}=0$

$\rho \frac{d \mathbf{v}}{d t}+\nabla \cdot \mathbf{P}=0$

$\rho \frac{d u}{d t}+\nabla \cdot \mathbf{q}+\mathbf{P}: \nabla \mathbf{v}=0$

$\frac{d n_{v_{1} v_{2}^{l} v_{3}}}{d t}+n_{v_{1} v_{2}^{l} v_{3}} \nabla \cdot \mathbf{v}+\nabla \cdot\left(n_{v_{1} v_{2}^{l} v_{3}} \mathbf{V}_{v_{1} v_{2}^{l} v_{3}}\right)=R_{v_{1} v_{2}^{l} v_{3}}^{v i b r}+R_{v_{1} v_{2}^{l} v_{3}}^{\text {react }}$,

$v_{m}=0, \ldots, L_{m}$,

$\frac{d n_{w}^{c}}{d t}+n_{w}^{c} \nabla \cdot \mathbf{v}+\nabla \cdot\left(n_{w}^{c} V_{c w}\right)=R_{c w}^{v i b r}+R_{c w}^{r e a c t}$,

$w=0, \ldots, L_{c}, \quad c=C O, O_{2}$,

$\frac{d n_{c}}{d t}+n_{c} \nabla \cdot \mathbf{v}+\nabla \cdot\left(n_{c} V_{c}\right)=R_{c}^{\text {react }}, \quad c=O, C$

where $\rho=\sum_{c} m_{c} n_{c}$ is the density, $m_{c}$ and $n_{c}$ are the mass and number density of species $c, P$ is the stress tensor, $u$ is the specific mixture energy, $q$ is the total heat flux, $\mathbf{V}_{v_{1} v_{2}^{l} v_{3}}$, $\mathbf{V}_{c w}$ are the diffusion velocities of vibrational states $\left(v_{1} v_{2}^{l} v_{3}\right)$ and $w, R_{v_{1} v_{2}^{l} v_{3}}^{v i b r}, R_{v_{1} v_{2}^{l} v_{3}}^{\text {react }}, R_{c w}^{v i b r}, R_{c w}^{\text {react }}$ are the stateto-state production terms due to vibrational transitions and chemical reactions, $R_{c}^{\text {react }}$ are the production rates of atoms $c, L_{m}$ is the number of excited vibrational levels in the $m$ th vibrational mode. 
The production terms in Eqs. (46)-(51) depend on the vibrational levels populations, number densities of species and rate coefficients of corresponding processes. The expressions for all production terms are given in [13]. The state-to-state transport theory for diatomic molecules was developed in [33] and generalized for polyatomic molecules in $[10,11]$. A particular feature of the heat transfer in the state-to-state approach is that the heat flux consists of four terms [34]

$$
\mathbf{q}=\mathbf{q}^{H C}+\mathbf{q}^{M D}+\mathbf{q}^{T D}+\mathbf{q}^{D V E}
$$

where the corresponding terms are responsible for heat conduction, mass and thermal diffusion, and diffusion of vibrational energy. Whereas the first three terms appear in the heat flux in all models (multi-temperature and one-temperature), the term $\mathbf{q}^{D V E}$ is specified only for the state-to-state kinetics. The heat conductivity in this case does not include the term connected to the vibrational energy, and the transport of vibrational energy is completely defined by the diffusion of vibrational states.

Since we are going to apply the state-to-state model to a $1 \mathrm{D}$ boundary layer problem, we simplify the fluid dynamics equations (46)-(51) using the boundary layer assumptions and Dorodnitsyn-Lees co-ordinate transformation in the vicinity of the stagnation point $[35,36]$. Thus we obtain a $1 \mathrm{D}$ boundary layer equation system in the state-to-state approach:

$\frac{\partial^{2} c_{v}}{\partial \eta^{2}}+f \operatorname{Sc} \frac{\partial c_{v}}{\partial \eta}=S_{v}, \quad v=1, \ldots, N u m S p$

$\frac{\partial^{2} \theta}{\partial \eta^{2}}+f \operatorname{Pr} \frac{\partial \theta}{\partial \eta}=S_{T}$

The direction $\eta$ corresponds to the normal to the surface in the self-similar coordinate system. The first NumSp equations are the species continuity equations whereas the last equation is the temperature one. As species we mean either molecules on the $v$-th vibrational level (for $\mathrm{CO}_{2}$, $v=\left(v_{1}, v_{2}, v_{3}\right)$, for diatomic species $v$ is the vibrational state) or atoms ( $v=\mathrm{C}$ or $\mathrm{O}) . c_{v}=\rho_{v} / \rho$ is the mass fraction of the $v$-species; $\theta=T / T_{e}$ is the ratio of the gas temperatures inside and at the external edge of the boundary layer; $f$ is the stream function, and Sc and Pr are the Schmidt and Prandtl numbers, respectively. The production terms $S_{v}$ and $S_{T}$ are given by expressions

$S_{v}=-\sum_{i=1}^{\text {NumSp }} B(v, i) c_{i}$

$S_{T}=\sum_{v=1}^{N u m S p} \frac{\mathrm{Le}}{C_{p} T_{e}} \varepsilon_{v} \sum_{i=1}^{\text {NumSp }} B(v, i) c_{i}$

where $C_{p}$ is the gas specific heat at constant pressure including, in the present model, only rotational and translational contributions, Le is the Lewis number, and the matrix $B(v, i)$ containing the kinetic terms is given in [11].

The implementation of the state-to-state description of mixtures containing $\mathrm{CO}_{2}$ to $\mathrm{CFD}$ is very computationally expensive. First, the number of equations for the vibrational level populations and number densities of atoms is equal to $N=L_{1} \times L_{2} \times L_{3}+L_{C O}+L_{O_{2}}+2=L_{\mathrm{CO}_{2}}+L_{C O}+L_{\mathrm{O}_{2}}+2$. For the $\mathrm{CO}_{2}$ molecule, the dissociation energy is $8.83859 .10^{-19} \mathrm{~J}$, which leads to the maximum vibrational quantum numbers 34, 67 and 20 for the symmetric, bending and asymmetric modes respectively, and the total number of $\mathrm{CO}_{2}$ states is 9018. Next problem is the calculation of transport coefficients. Thus the number of diffusion coefficients about $N^{2}$, and the dimension of transport linear systems which should be solved at each step of numerical solution is of the order of $N$.

Based on the above arguments, we propose to use in the fluid dynamic equations a simplified transport model with constant Prandtl and Schmidt numbers to obtain preliminary results on $\mathrm{CO}_{2}$ vibrational kinetics. Then, in order to evaluate the influence of vibrational kinetics on the transport terms, we use the macroscopic gas parameters obtained numerically solving the fluid dynamics equations and the rigorous transport model for the calculation of the transport coefficients and heat flux. Such a post-processing procedure is certainly approximate, however, the main trends and contributions of various processes to the heat transfer can be qualitatively estimated.

Another way of studying vibrational non-equilibrium in polyatomic viscous gases is to use advanced multitemperature models for kinetics and transport properties taking into account real gas effects and different rates of vibrational exchanges.

\section{MULTI-TEMPERATURE MODELS FOR $\mathrm{CO}_{2}$ MIXTURES}

In this section we consider multi-temperature models for the flows of the five-component reacting mixture $\mathrm{CO}_{2} / \mathrm{CO} /$ $\mathrm{O}_{2} / \mathrm{C} / \mathrm{O}$ near spacecrafts entering the Mars atmosphere. The multi temperature description of non-equilibrium kinetics in reacting mixtures is much simpler compared to the state-tostate model considered in the previous section and therefore more suitable for applications in computational fluid dynamics. Actually, in multi-temperature approaches fluid dynamic equations are coupled to relaxation equations for vibrational temperatures of different molecular species instead of equations for vibrational level populations of different molecular components. The multi-temperature models are based on the experimental and theoretical data on relaxation times of different processes in the considered mixtures. The analysis of these data for $\mathrm{CO}_{2}$ containing mixtures under high temperature condition shows that the translational and rotational relaxation as well as $\mathrm{CO}_{2}$ intramode vibrational energy exchanges proceed much faster compared to other vibrational energy transitions and chemical reactions. Such relations between characteristic times make it possible to introduce vibrational temperatures $T_{m}(m=1,2,3)$ for three vibrational $\mathrm{CO}_{2}$ modes and express vibrational level populations in terms of these temperatures. Consequently, the number of governing equations is essentially reduced: instead of $L_{\mathrm{CO} 2}$ equations for level populations one may consider only three equations for vibrational temperatures. 
Experimental data also show that the inter-mode exchange between $\mathrm{CO}_{2}$ symmetric and bending modes (11) proceeds fast due to the Fermi resonance between the frequencies of these modes and can be considered as a rapid process along with the intra-mode energy exchanges. The remaining kinetic processes are slow and have relaxation times of the same order as the mean time of the gas-dynamic parameters variation. In this case the relaxation times of different processes in the considered mixture satisfy the following conditions:

$\tau_{t r}<\tau_{\text {rot }}<\tau_{V V_{m}} \sim \tau_{V V_{1-2}}<<\tau_{V T_{2}} \sim \tau_{V V_{2-3}} \sim \tau_{V V_{1-2-3}}<\tau_{\text {react }} \sim \theta$,

$m=1,2,3$.

The rapid $\mathrm{V}_{1-2}$ exchange makes it possible to consider the coupled (symmetric-bending) mode with a single vibrational temperature $T_{12}$. For the case of harmonic oscillators we represent the vibrational distributions of $\mathrm{CO}_{2}$ molecules as a combination of two non-equilibrium Boltzmann distributions with the vibrational temperature $T_{12}$ in the coupled mode and $T_{3}$ in the asymmetric mode.

$$
\begin{aligned}
& n_{v_{1} v_{2} v_{3}}\left(T_{12}, T_{3}\right)=\frac{n_{C O_{2}}\left(v_{2}+1\right)}{Z_{v i b r}^{C O_{2}}\left(T_{12}, T_{3}\right)} \\
& \exp \left(-\frac{\left(2 v_{1}+v_{2}\right) \varepsilon_{010}}{k T_{12}}-\frac{v_{3} \varepsilon_{001}}{k T_{3}}\right),
\end{aligned}
$$

where $Z_{v i b r}^{\mathrm{CO}_{2}}\left(T_{12}, T_{3}\right)$ is the non-equilibrium $\mathrm{CO}_{2}$ vibrational partition function. In the present study, the diatomic species $\mathrm{CO}$ and $\mathrm{O}_{2}$ are supposed to equilibrate faster than $\mathrm{CO}_{2}$ and are considered in thermal equilibrium. The vibrational nonequilibrium excitation of $\mathrm{CO}$ and $\mathrm{O}_{2}$ species in considered mixture is taken into account in [13].

The governing equations are derived in [15] from the kinetic equations for distribution functions and include the conservation equations of mass, momentum and total energy coupled to the equations of chemical kinetics and two relaxation equations for vibrational energies of coupled and asymmetric modes:

$\frac{d \rho}{d t}+\rho \nabla \cdot \mathbf{v}=0$,

$\rho \frac{d v}{d t}+\nabla \cdot \mathbf{P}=0$

$\rho \frac{d u}{d t}+\nabla \cdot \mathbf{q}+\mathbf{P}: \nabla \mathbf{v}=0$,

$\frac{d n_{c}}{d t}+n_{c} \nabla \cdot \mathbf{v}+\nabla \cdot\left(n_{c} \mathbf{V}_{c}\right)=R_{c}^{\text {react }}, \quad c=\mathrm{CO}_{2}, \mathrm{CO}, \mathrm{O}_{2}, \mathrm{C}, \mathrm{O}$,

$\rho_{\mathrm{CO}_{2}} \frac{d E_{12}}{d t}+\nabla \cdot \mathbf{q}_{12}=R_{12}-m_{\mathrm{CO}_{2}} E_{12} R_{\mathrm{CO}_{2}}^{\text {react }}+E_{12} \nabla \cdot\left(\rho_{\mathrm{CO}_{2}} \mathbf{V}_{\mathrm{CO}_{2}}\right)$,

$\rho_{\mathrm{CO}_{2}} \frac{d E_{3}}{d t}+\nabla \cdot \mathbf{q}_{3}=R_{3}-m_{\mathrm{CO}_{2}} E_{3} R_{\mathrm{CO}_{2}}^{\text {react }}+E_{3} \nabla \cdot\left(\rho_{\mathrm{CO}_{2}} \mathbf{V}_{\mathrm{CO}_{2}}\right)$.

Here $E_{12}\left(T_{12}\right), \quad E_{3}\left(T_{3}\right)$ are the specific vibrational energies of the coupled and asymmetric modes, respectively, $\mathbf{q}_{12}, \quad \mathbf{q}_{3}$ are the fluxes of vibrational energy in the corresponding modes. The relaxation terms $R_{c}^{\text {react }}, R_{12}, R_{3}$ describe slow processes which include dissociation, recombination, exchange reactions, inter-mode $\mathrm{CO}_{2}$ exchanges except $\mathrm{VV}_{12}$ exchange and $\mathrm{VT}_{2}$ transitions.

The transport and relaxation terms in Eqs. (59)-(64) are reported in details in [13] on the basis of the generalized Chapman-Enskog method. All transport coefficients are found from linear transport systems given in [12, 13], elements of these systems depend on the cross sections of the most often collisions with inelastic non-resonant energy transitions. The expressions for heat fluxes $\mathbf{q}, \mathbf{q}_{12}$, and $\mathbf{q}_{3}$ contain gradients of temperatures $T, T_{12}, T_{3}$ and heat conductivity coefficients for transfer of total energy and vibrational energies of coupled and asymmetric $\mathrm{CO}_{2}$ modes.

The relaxation terms $R_{c}^{\text {react }}$ specify processes (19)-(24). These expressions involve three-temperature rate coefficients for $\mathrm{CO}_{2}$ dissociation $k_{\mathrm{CO}_{2}, \text { diss }}^{M}\left(T, T_{12}, T_{3}\right)$ and exchange reactions as well as thermal equilibrium reaction rate coefficients for diatomic molecules that depend only on the gas temperature. The expressions for three-temperature $\mathrm{CO}_{2}$ reaction rate coefficients are obtained by averaging the statedepending rate coefficients considered in Section 3 with vibrational distributions (58). The rate coefficient for $\mathrm{CO}_{2}$ dissociation is written in the form derived in [12] using the generalization of the Treanor-Marrone model [27]:

$$
\begin{aligned}
& k_{C O_{2}, d i s s}^{M}\left(T, T_{12}, T_{3}\right)=k_{d i s s}^{e q, M} \frac{Z_{v i b r}^{C O_{2}}(T)}{Z_{v i b r}^{C O_{2}}(-U) Z_{v i b r}^{C O_{2}}\left(T_{12}, T_{3}\right)} \times \\
& \times \sum_{v_{1}, v_{2}, v_{3}}\left(v_{2}+1\right) \exp \left(\begin{array}{l}
\frac{v_{1} \varepsilon_{100}+v_{2} \varepsilon_{010}}{k}\left(\frac{1}{T}-\frac{1}{T_{12}}\right)+ \\
\frac{v_{3} \varepsilon_{001}}{k}\left(\frac{1}{T}-\frac{1}{T_{3}}\right)+\frac{\varepsilon_{v_{1} v_{2} v_{3}}}{k U}
\end{array}\right) .
\end{aligned}
$$

Rate coefficients for backward reactions are found using the detailed balance principle. The approximate expressions for production terms of slow processes (7), (12) and (13) via relaxation times which can be measured experimentally are proposed in [13].

Thus we derived self-consistent macroscopic flow descriptions in the state-to-state and multi-temperature approaches. These governing equations are solved numerically in the next section for particular test cases.

\section{RESULTS AND DISCUSSION}

Let us consider first the results of multi-temperature modelling. Eqs. (59)-(64) with rigorous kinetic schemes for transport coefficients have been solved numerically for a flow in a $2 \mathrm{D}$ viscous shock layer near the blunt bodies imitating the form of the spacecrafts MSRO (Mars Sample Return Orbiter) and Mars Express for two test cases: $V_{\infty}=5223 \mathrm{~m} / \mathrm{s}, \rho_{\infty}=2.93 \cdot 10^{-4} \mathrm{~kg} / \mathrm{m}^{3}, T_{\infty}=140 \mathrm{~K}(\mathrm{TC} 1)$ and $V_{\infty}=5687 \mathrm{~m} / \mathrm{s}, \quad \rho_{\infty}=3.141 \cdot 10^{-5} \mathrm{~kg} / \mathrm{m}^{3}, T_{\infty}=140 \mathrm{~K}$ (TC2). The orthogonal system of co-ordinates $(s, n)$ is used, where $s$ is counted from the stagnation point along the body 

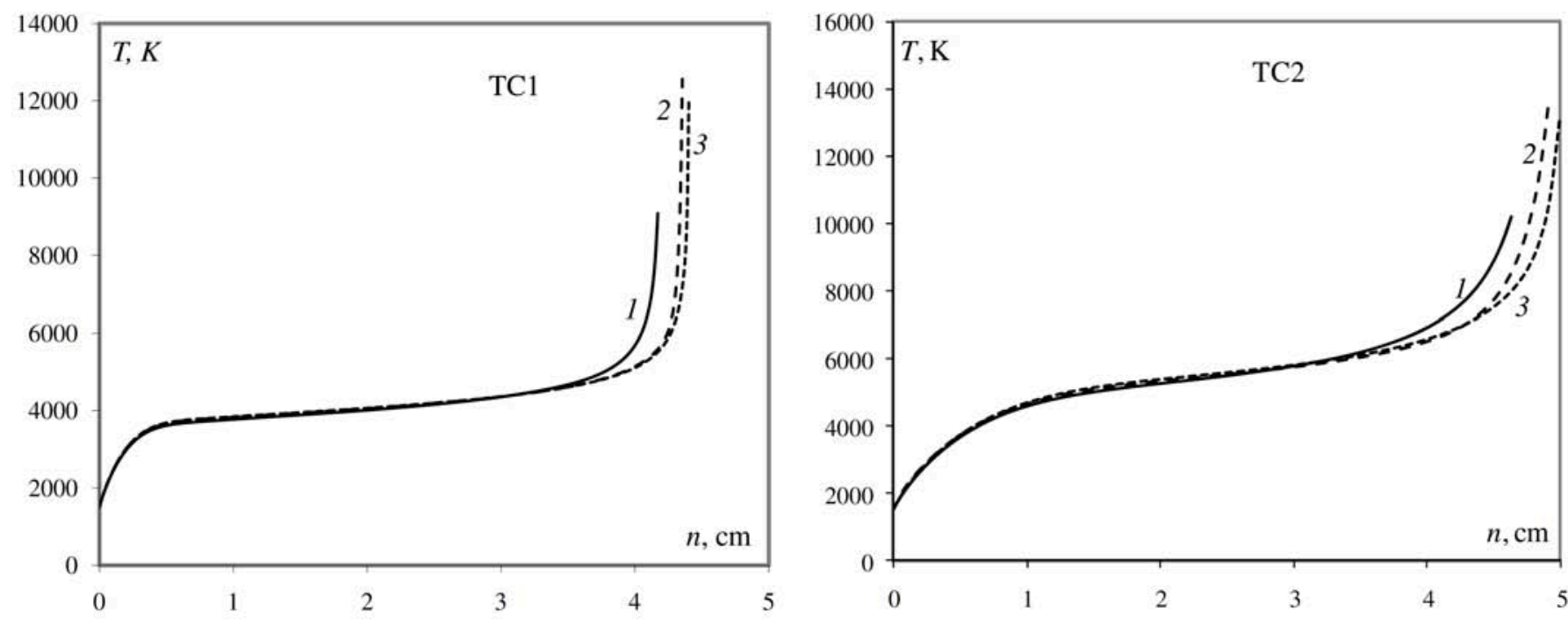

Fig. (2). Gas temperature along the stagnation line. (a) TC1: $V_{\infty}=5223 \mathrm{~m} / \mathrm{s}, \rho_{\infty}=2.93 \cdot 10^{-4} \mathrm{~kg} / \mathrm{m}^{3}, T_{\infty}=140 \mathrm{~K},(\mathbf{b}) \mathrm{TC} 2: V_{\infty}=5687 \mathrm{~m} / \mathrm{s}$, $\rho_{\infty}=3.141 \cdot 10^{-5} \mathrm{~kg} / \mathrm{m}^{3}, T_{\infty}=140 \mathrm{~K}$. Curve 1: one-temperature model; curve 2: three-temperature model; curve 3: two-temperature model $[1]$.

surface while $n$ is normal to the surface. The free stream is supposed to be consisted of pure $\mathrm{CO}_{2}$, the body surface is assumed to be either non-catalytic or fully catalytic. The results are presented in $[14,15]$ and show the behaviour of flow parameters in a shock layer and heat transfer to the vehicle surfaces.

In order to understand the influence of a kinetic model on macroscopic flow parameters and heat transfer to the body surface, we solved in $[14,15]$ the system of governing equations not only in the three-temperature approximation but also the two-temperature and one-temperature approaches. In the first case, a single vibrational temperature for all three $\mathrm{CO}_{2}$ modes $T_{v}=T_{12}=T_{3}$ is introduced; and in the second model $T_{12}=T_{3}=T$.

The profiles of the gas temperature along the stream line orthogonal to the surface in the shock layer and the heat flux

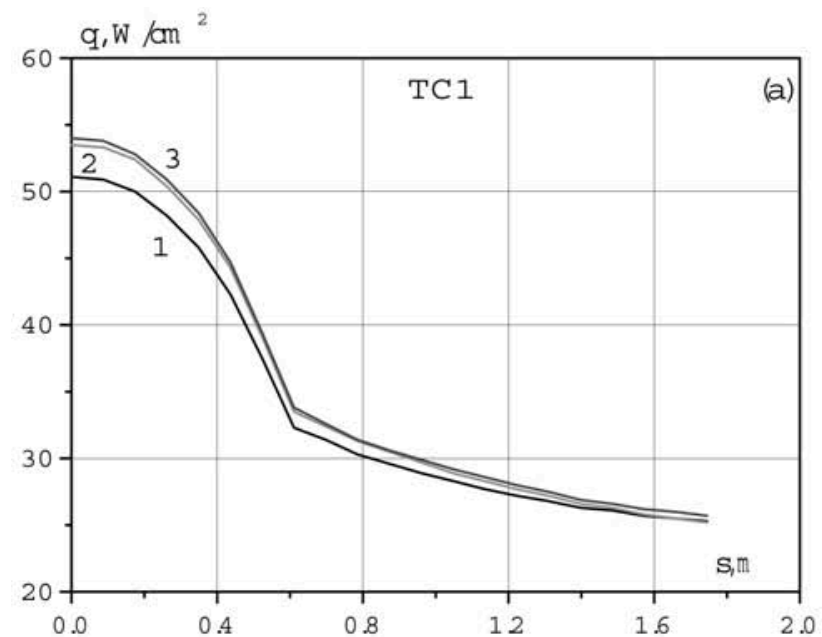

values on the body surface obtained in $[14,15]$ for the spacecraft MSRO are presented in Figs. $(\mathbf{2}, \mathbf{3})$. The values $n=0$ and $s=0$ correspond to the stagnation point. Curves 1 correspond to the one-temperature approach, 2 to the threetemperature approach, and 3 to the two-temperature approach.

The results show that the profiles of gas temperature corresponding to the two- and three-temperature models (curves 2,3) differ rather weakly, the discrepancy does not exceed $5 \%$. This justifies the validity of the approximate two-temperature model under the entry conditions. On the other hand, far from the surface $(n>3 \mathrm{~cm})$ one can notice considerable difference between the results obtained for thermal equilibrium (one-temperature) and non-equilibrium (multi-temperature) flows. The vibrational temperatures $T_{12}$ and $T_{3}$ equilibrate coming to the surface as well as the specific vibrational energies. The difference between the

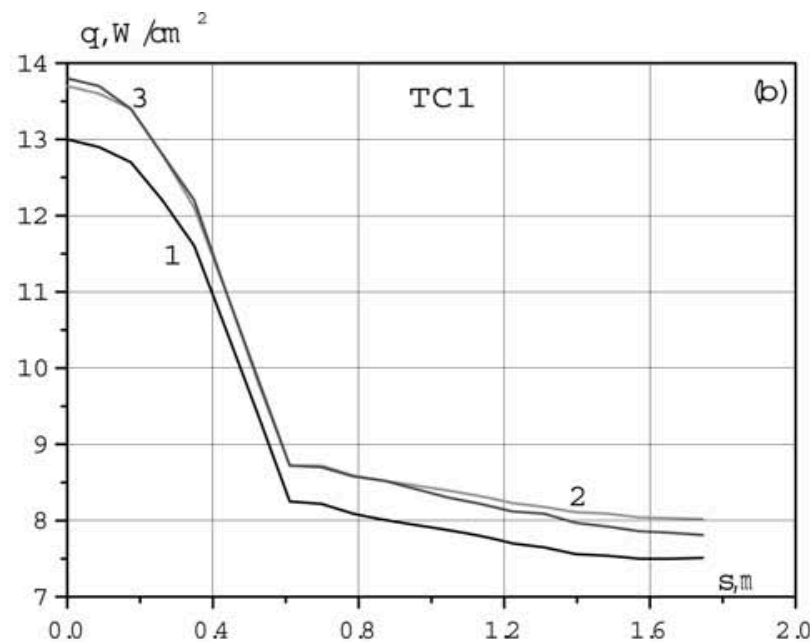

Fig. (3). Heat flux along the body surface, TC1: $V_{\infty}=5223 \mathrm{~m} / \mathrm{s}, \rho_{\infty}=2.93 \cdot 10^{-4} \mathrm{~kg} / \mathrm{m}^{3}, T_{\infty}=140 \mathrm{~K}$. (a) Catalytic surface; (b) Non-catalytic surface. 
values of the total vibrational energy found in the threetemperature, two-temperature and one-temperature approaches is very small near the surface. Non-equilibrium vibrational $\mathrm{CO}_{2}$ excitation leads to a slight increase in distance between the shock wave and body surface, noticeable rising (up to $30 \%$ ) of the gas temperature near the shock front and weakly affects the gas temperature near the surface. Vibrational non-equilibrium of $\mathrm{CO}_{2}$ molecules also leads to the increase of the surface heat flux up to $10 \%$. The results were obtained using three models for thermal equilibrium reaction rates [29, 30-32]. While only the slight influence of chemical reaction model on the heat transfer to the non-catalytic surface was found, near the fully catalytic surface this effect occurs more essential [15]. Recently a strong influence of a body shape on the gas parameters in a shock layer and heat fluxes to the surface was found in [37].

Now let us consider the state-to-state solution of a 1D boundary layer problem (see Eqs. (53) and (54)). In the test cases considered below, we use as external edge boundary conditions the macroscopic gas flow parameters obtained in the frame of the TC2 multi-temperature test case discussed above. We choose the conditions corresponding to the region where thermal equilibrium establishes in the shock layer, i.e. about $n=3 \mathrm{~cm}$ (see Fig. $\mathbf{2 b}$ ). Note that at this distance the mixture composition is still far from chemical equilibrium. Thus we set $\left(c_{\mathrm{CO}_{2}}\right)_{e}=10.4 \%,\left(c_{\mathrm{CO}}\right)_{e}=57.6 \%,\left(c_{C}\right)_{e}=0.3 \%$, $\left(c_{O_{2}}\right)_{e}+\left(c_{o}\right)_{e}=31.7 \%, T_{e}=6000 \mathrm{~K}, p_{e}=888 \mathrm{Nm}^{-2}$, and $\beta=2708 \mathrm{~s}^{-1}$, where $T_{e}$ and $p_{e}$ are temperature and pressure at the boundary layer edge, and $\beta$ is the gradient of the free stream velocity with respect to the coordinate along the surface (its physical meaning is the inverse of the residence time of a fluid element in a particular point of the boundary layer). The vibrational distributions at the external edge are

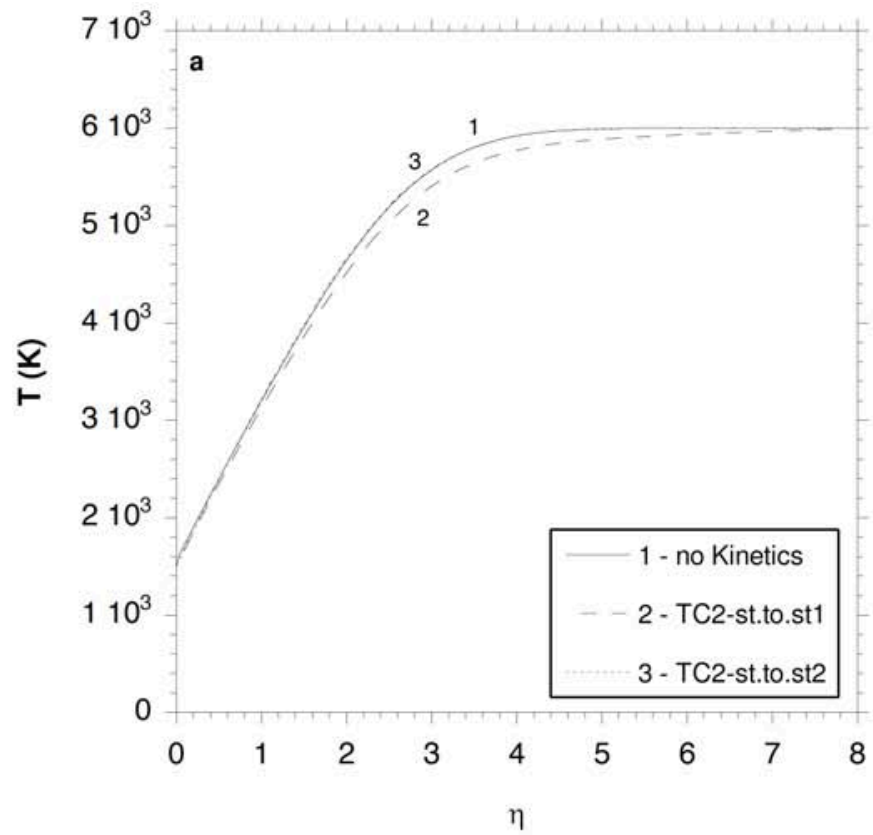

Boltzmann at $T=T_{e}$ since we consider thermal equilibrium region. At the surface, the temperature $T_{w}=1500 \mathrm{~K}$, and the surface is assumed non-catalytic, so that the gradients of species molar fractions are zero.

Two test cases are considered with simplified kinetic schemes. In the first test case (TC2-st.to.st1), the following processes are taken into account: (7), (11), (13), (15), (16), (19). In the second test case (TC2-st.to.st2), only vibrational exchanges (7), (11), (13) in $\mathrm{CO}_{2}$ molecules are included; dissociation, recombination and inter-molecular transitions are neglected. Although simplified, these kinetic schemes describe the most important channels of vibrational relaxation and thus can describe the main features of $\mathrm{CO}_{2}$ kinetics. Moreover, in order to minimize computational costs, a reduced number of $\mathrm{CO}_{2}$ vibrational levels is considered by setting the $\mathrm{CO}_{2}$ dissociation energy to 4.8065.10 $0^{-19} \mathrm{~J}$ (see [11] for details); in this way the maximum vibrational quantum numbers become 17,33 and 9 in the symmetric, bending and asymmetric modes respectively, resulting in the total number of $\mathrm{CO}_{2}$ states of 1224 , which is much less than in the full model. In [11] it is shown that such a reduced model practically does not affect the flow parameters for a non-catalytic surface.

In Fig. (4) the temperature profile (Fig. 4a) and the total vibrational energy per mass unit (Fig. 4b) across the boundary layer are shown. Curve 1 corresponds to the solution of the energy equation (54) without the production term. Solving Eq. (54) with $S_{T}=0$ we find the temperature at each grid step, and then assume that the vibrational distributions are Boltzmann with the local temperature. Curve 2 corresponds to the TC2-st.to.st1 test case and curve 3 to the TC2-st.to.st2 test case. One can see that vibrational transitions do not affect the temperature, whereas dissociation and recombination processes slightly modify the

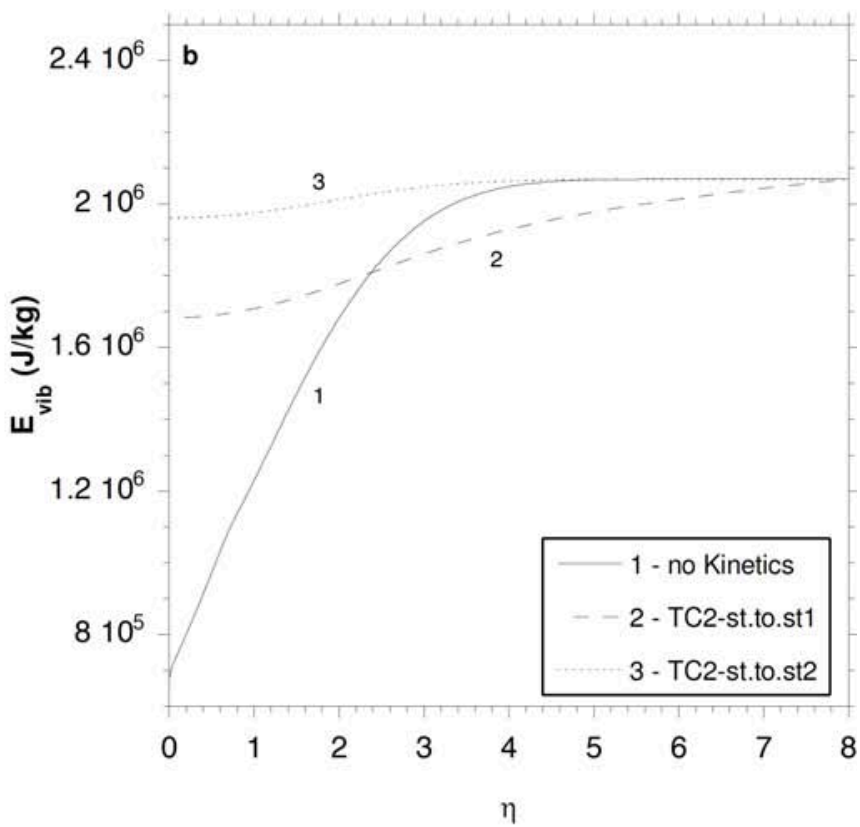

Fig. (4). Temperature profile (a) and total vibrational energy per mass unit (b) across the boundary layer. Comparison of different kinetic schemes: no kinetics (curve 1); processes (7), (11), (13), (15), (16), and (19) are included (curve 2); processes (7), (11), and (13) are included (curve 3). 

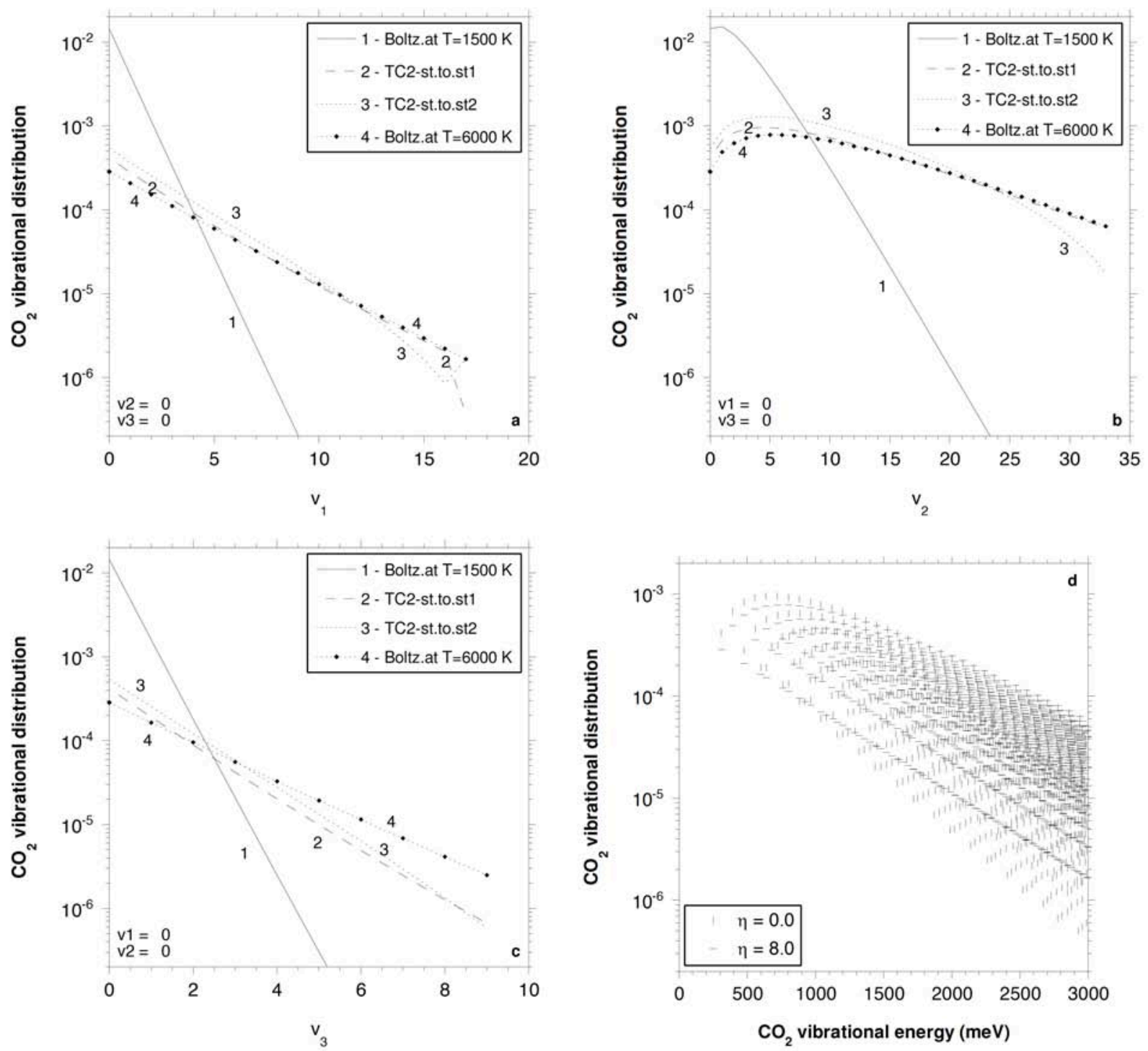

Fig. (5). Vibrational distributions versus each mode quantum number at fixed values of the other two modes quantum numbers (a-c); whole distributions versus the vibrational energy for the TC2-st.to.st1 test case (d). Case TC2-st.to.st1: processes (7), (11), (13), (15), (16), and (19) are included; Case TC2-st.to.st2: processes (7), (11), and (13) are included.

temperature profile at $1<\eta<7$. The total vibrational energy strongly depends on the vibrational distribution, especially close to the surface. Assuming Boltzmann vibrational distributions in the flow leads to considerable underestimation of the vibrational energy at the surface. This effect can be of importance near the catalytic wall, where the transport of vibrational energy gives noticeable contribution to the energy flux.

The vibrational distributions versus quantum numbers of each mode, at fixed values of the other two modes, are plotted on Fig. (5a-c). Curve 1 corresponds to the equilibrium Boltzmann distribution at $T=T_{w}$, curve 2 corresponds to the distribution on the surface $(\eta=0)$ obtained under conditions of TC2-st.to.st1, curve 3 corresponds to the distribution on the surface $(\eta=0)$ obtained in the TC2-st.to.st2 case, finally curve 4 is the external edge vibrational distribution (the Boltzmann distribution at $T_{e}$ ). The considered processes influence the flow, which does not remain frozen in the boundary layer; however the flight conditions do not give to the kinetic processes the time to thermalize the flow, so that it reaches the surface far from local equilibrium: curves 2 and 3 of Figs. (5a-c) do not coincide with curve 1 . The statement that the flow does not remain frozen in the boundary layer is also evident in Fig. (5d), that gives an idea of the vibrational distributions as functions of vibrational energy (and not fixed quantum numbers) on the surface and at the external edge for the TC2-st.to.st1 test case: the two distributions do not coincide. It is worth mentioning that the multi-temperature models give thermal equilibrium vibrational distributions near the surface (see also Fig. 2).

In Fig. (6) the heat flux normal to the surface is reported. Curve 1 corresponds to the calculation neglecting kinetics, curve 2 to the TC2-st.to.st1 test case, and curve 3 to the TC2-st.to.st2 test case. When no kinetics is included in the 
calculation, the heat flux on the surface is slightly higher. Comparing curves 2 and 3 corresponding to more adequate calculations we conclude that including dissociation, recombination and inter-molecular transitions does not influence noticeably the heat flux; however it would be important to include all the state-to-state kinetic processes (1)-(24) to give more accurate evaluations. Besides that we expect more important effect of vibrational kinetics in the case of a catalytic wall, when recombination pumps vibrationally excited states, and contributions of mass and vibrational energy diffusion to the heat flux become greater.

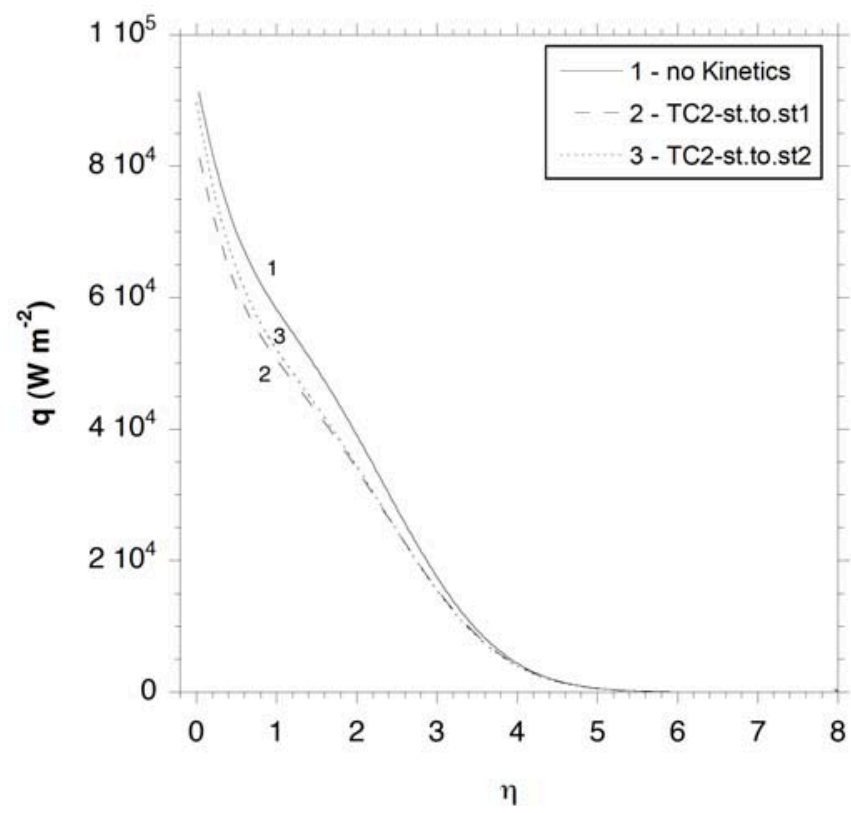

Fig. (6). Heat flux versus the normal to the surface. Comparison of different kinetic schemes: no kinetics (curve 1); processes (7), (11), (13), (15), (16), and (19) are included (curve 2); processes (7), (11), and (13) are included (curve 3).

Now let us compare the vibrational energy and the heat flux on the surface obtained in the multi-temperature and state-to-state approaches for the TC2. In Table $\mathbf{4}$ the specific vibrational energy and the total heat flux calculated at $\eta=0$ are given. The specific vibrational energy obtained in the state-to-state approach is considerably higher than that found using the multi-temperature or one-temperature model, which is rather close to $E_{v i b r}$ calculated neglecting vibrational kinetics. This confirms again that the state-tostate flow is not in vibrational equilibrium close to the surface, and the store of vibrational energy is higher than in the multi-temperature flows. Nevertheless, it does not affect the total heat flux. In the multi-temperature model, the heat flux was calculated using three chemical reaction models. In the state-to-state simulation, the Mc-Kenzie model [29] was applied for the thermal equilibrium dissociation rate coefficients. One can see that the heat fluxes calculated in the multi-temperature and state-to-state models using the same chemical reaction rates agree very well. The influence of the detailed kinetics on the energy transfer is found to be negligible for a non-catalytic surface. Once again, it is worth mentioning that for a catalytic surface, the contribution of state-to-state kinetics to the heat flux can be much more important. These effects are the subject of our future study.

\section{CONCLUSION}

A comprehensive kinetic scheme indicating all possible mechanisms of vibrational relaxation and chemical reactions is proposed for $\mathrm{CO}_{2}$ flows. Detailed information on the rate coefficients of processes characteristic for Mars entry problems is presented. State-to-state and multi-temperature approaches are developed for the prediction of nonequilibrium gas flow parameters and heat transfer. The accurate state-to-state model is applied for modelling of a 1D boundary layer near the spacecraft entering the Mars atmosphere whereas the quasi-stationary approach is used to study a 2D shock layer between the shock front and MSRO surface. The state specific model is unfortunately very time consuming. Even reducing the number of vibrational states, we obtain 1224 equations for $\mathrm{CO}_{2}$ vibrational level populations coupled to fluid dynamic equations.

Table 4. Specific vibrational energy and heat flux at $\eta=0$

for TC2 Case: $V_{\infty}=5687 \mathrm{~m} / \mathrm{s}, \quad \rho_{\infty}=3.141 \cdot 10^{-5}$ $\mathrm{kg} / \mathrm{m}^{3}, T_{\infty}=140 \mathrm{~K}$. Case TC2-st.to.st1: processes (7), (11), (13), (15), (16), and (19) are included; case TC2-st.to.st2: processes (7), (11), and (13) are included.

\begin{tabular}{|c|c|c|c|c|}
\hline & MT, TC2 & $\begin{array}{c}\text { STS, } \\
\text { TC2-st.to.st1 }\end{array}$ & $\begin{array}{c}\text { STS, } \\
\text { TC2-st.to.st2 }\end{array}$ & $\begin{array}{c}\text { No } \\
\text { Kinetics }\end{array}$ \\
\hline \hline$E_{\text {vibr }, \mathrm{MJ} / \mathrm{kg}}$ & 0.65 & 1.66 & 1.93 & 0.71 \\
\hline \multirow{2}{*}{$q, \mathrm{~kW} / \mathrm{m}^{2}$} & $71.1[30]$ & & & \\
\cline { 2 - 5 } & $96.0[29]$ & 83.54 & 89.68 & 91.3 \\
\cline { 2 - 5 } & $80.5[32]$ & & & \\
\hline
\end{tabular}

The results obtained on the basis of multi-temperature and one-temperature models show the strong influence of non-equilibrium vibrational excitation just behind a shock on the gas temperature and specific vibrational energy. However, near the surface non-equilibrium effects diminish, the difference between the values of gas temperature and vibrational temperature found in three-temperature and twotemperature non-equilibrium models becomes very small. Thus we conclude that multi-temperature models give thermal equilibrium reacting flows near the surface.

This is not the case for the state-resolved vibrational mechanism. The results show that vibrational distributions near the surface are far from the local vibrational and chemical equilibrium, and a noticeable difference between the values of $\mathrm{CO}_{2}$ surface specific vibrational energies obtained in the state-to-state and quasi-stationary approaches is found. At the same time, a weak influence of a kinetic model on the heat transfer to the non-catalytic vehicle surface is shown for all considered approaches. For the catalytic surface, we expect more important effects of nonequilibrium kinetics on the heat flux.

\section{CONFLICT OF INTEREST}

The authors confirm that this article content has no conflict of interest. 


\section{ACKNOWLEDGEMENTS}

The research leading to these results has received funding from the European Community's Seventh Framework Programme (FP7/2007-2013) under grant agreement no. 242311 and Russian foundation for basic research (projects 11-01-00408 and 12-08-00826). The authors are grateful to Dr. N. G. Syzranova for providing data on the used thermal equilibrium reaction rates and numerical results obtained in the quasi-stationary approaches.

\section{REFERENCES}

[1] Taylor R, Bitterman S. Survey of vibrational relaxation data for process important in the $\mathrm{CO}_{2}-\mathrm{N}_{2}$ laser system. Rev Mod Phys 1969; 41(1): 26-47.

[2] Thomson R. The thermal conductivity of gases with vibrational internal energy. J Phys D 1978; 11: 2509.

[3] Brun R. Transport properties in reactive gas flows. AIAA Paper. 1988; pp. 88-2655.

[4] Capitelli M, Bruno D, Laricchiuta A. Fundamental aspects of plasma chemical physics: transport. springer series on atomic, optical, and plasma physics. Berlin: Springer Verlag 2013; vol. 74.

[5] Likalter A. On the vibrational distribution of polyatomic molecules. Prikl Mekh Tekn Fiz 1976; 4: 3. (In Russian)

[6] Cenian A. Study of non-equilibrium vibrational relaxation of $\mathrm{CO}_{2}$ molecules during adiabatic expansion in a supersonic nozzle. The Treanor distribution - existence and generation. Chem Phys 1989; 132(1-2): 41-8.

[7] Kustova E, Nagnibeda E. Non-equilibrium distributions in $\mathrm{CO}_{2}$ and their influence on the transport and thermodynamic properties. Rarefied Gas Dyn 1999; 2: 289-96.

[8] Chikhaoui A, Kustova E. Effect of strong excitation of $\mathrm{CO}_{2}$ asymmetric mode on transport properties. Chem Phys 1997; 216(3): 297-315.

[9] Kustova E, Nagnibeda E, Chikhaoui A. Heat transfer and diffusion in mixtures containing $\mathrm{CO}_{2}$. AIP Conf Proc 2003; 663: 100-5.

[10] Kustova E, Nagnibeda E. State-to-state theory of vibrational kinetics and dissociation in three-atomic gases. AIP Conf Proc 2001; 585: 620-7.

[11] Armenise I, Kustova E. State-to-state models for $\mathrm{CO}_{2}$ molecules: from the theory to an application to hypersonic boundary layers. Chem Phys 2013; 415: 269-81.

[12] Kustova E, Nagnibeda E. On a correct description of a multitemperature dissociating $\mathrm{CO}_{2}$ flow. Chem Phys 2006; 321(6): 293310.

[13] Kustova E, Nagnibeda E. Kinetic model for multi-temperature flows of reacting carbon dioxide mixture. Chem Phys 2012; 398(1): $111-7$.

[14] Kustova E, Nagnibeda E, Shevelev Y, Syzranova N. Nonequilibrium supersonic $\mathrm{CO}_{2}$ flows with real gas effects near a blunt body. AIP Conf Proc 2009; 1084: 831-6.

[15] Kustova E, Nagnibeda E, Shevelev Y, Syzranova N. Comparison of different models for non-equilibrium $\mathrm{CO}_{2}$ flows in a shock layer near a blunt body. Shock Waves 2011;21(3): 273-87.

[16] Herzberg G, Infrared and raman spectra of polyatomic molecules. New York: D. Van Nostrand Company, Inc. 1951.

[17] Makarov V, Losev S. Application of the database of physicalchemical processes for the creation of the equations set for gases with chemical reactions and vibrational relaxation. Khim Phizika 1997; 16(5): 29-43.

[18] Borrell P, Millward G. Vibrational energy transfer process in shock-heated binary gas mixtures of $\mathrm{CO}$ with $\mathrm{CO}_{2}, \mathrm{~N}_{2} \mathrm{O}$ and COS. J Chem Phys 1972; 57(1): 462.

[19] Salnikov V, Starik A. Numerical analysis of energetic characteristics of gas-dynamic lasers on the hydrocarbon fuels combustion products. Teplofizika Vysokikh Temp 1995; 33(1): 121.

[20] Achasov O, Ragosin D. Rate constants of $\mathrm{V}-\mathrm{V}$ exchange for $\mathrm{CO}_{2-}$ GDL. Preprint 16. Minsk, Bielarus: Institute of Heat and Mass Transfer 1986.

[21] Schwartz RN, Slawsky ZI, Herzfeld KF. Calculation of vibrational relaxation times in gases. J Chem Phys 1952; 20(10): 1591.

[22] Blauer J, Nickerson G. A survey of vibrational relaxation rate data for processes important to $\mathrm{CO}_{2}-\mathrm{N}_{2}-\mathrm{H}_{2} \mathrm{O}$ infrared plume radiation. AIAA Paper 1974; pp. 74-536.

[23] Billing GD. Semiclassical calculation of energy transfer in polyatomic molecules. II. The effect of anharmonic coupling terms. Chem Phys 1980; 46(1-2): 123-31.

[24] Clary D. Ab initio calculations of vibrational relaxation rate coefficients for the collisions of $\mathrm{CO}_{2}$ with helium and neon atoms Chem Phys 1982; 65(2): 247-57.

[25] Wickham-Jones C, Simpson C, Clary D. Experimental and theoretical determination of rate constants for vibrational relaxation of $\mathrm{CO}_{2}$ and $\mathrm{CH}_{3} \mathrm{~F}$ by He. Chem Phys 1987; 117(1): 9-16.

[26] Bartolomei $\mathrm{M}$, Pirani $\mathrm{F}$, Laganà $\mathrm{A}$, Lombardi $\mathrm{A}$. A full dimensional grid empowered simulation of the $\mathrm{CO}_{2}+\mathrm{CO}_{2}$ processes. J Comp Chem 2012; 33(22): 1806-19.

[27] Marrone P, Treanor C. Chemical relaxation with preferential dissociation from excited vibrational levels. Phys Fluids 1963; 6(9): 1215

[28] Aliat A. State-to-state dissociation--recombination and chemical exchange rate coefficients in excited diatomic gas flows. Phys A 2008; 387(16-17): 4163-82.

[29] McKenzie RL, Arnold JO. Experimental and theoretical investigation of the chemical kinetics and non-equilibrium $\mathrm{CN}$ radiation behind shock waves in $\mathrm{CO}_{2}-\mathrm{N}_{2}$-mixtures. AIAA Paper 1967: pp. 67-322.

[30] Park C, Howe JT, Jaffe RL, Candler GV. Review of chemicalkinetic problems of future NASA missions, II: Mars entries. J Thermophys Heat Transf 1994: 8(1): 9-23.

[31] Ibragimova L, Smekhov G, Shatalov O. Dissociation rate constants of diatomic molecules under thermal equilibrium conditions. Fluid Dyn 1999; 34(1): 153-7.

[32] Iragimova LB. Rate coefficients of chemical reactions in the hightemperature gas $\mathrm{CO}_{2}$. Math Model 2000; 12(4): 3-19.

[33] Kustova E, Nagnibeda E. Transport properties of a reacting gas mixture with strong vibrational and chemical non-equilibrium. Chem Phys 1998; 233(1): 57-75.

[34] Kustova E, Nagnibeda E, Alexandrova T, Chikhaoui A. On the non-equilibrium kinetics and heat transfer in nozzle flows. Chem Phys 2002; 276(2): 139-54.

[35] Anderson J. Hypersonic and high temperature gas dynamics. New York: McGraw-Hill 1989.

[36] Capitelli M, Armenise I, Gorse C. State-to-state approach in the kinetics of air components under re-entry conditions. J Thermophys Heat Transf 1997; 11(4): 570-8.

[37] Kustova E, Nagnibeda E, Shevelev Y, Syzranova N. The influence of $\mathrm{CO}_{2}$ kinetics on the hypersonic flow near blunt bodies. AIP Conf Proc 2012; 1501: 1102-9.

This is an open access article licensed under the terms of the Creative Commons Attribution Non-Commercial License (http://creativecommons.org/licenses/by-nc/3.0/) which permits unrestricted, non-commercial use, distribution and reproduction in any medium, provided the work is properly cited. 